\title{
Hair and nail nicotine levels of mothers and their infants as valid biomarkers of exposure to intrauterine tobacco smoke
}

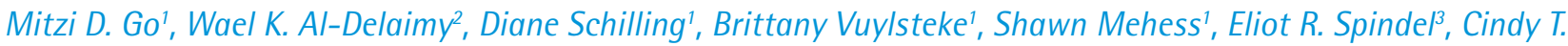 \\ McEvoy
}

\begin{abstract}
INTRODUCTION Tobacco use remains the single most modifiable cause of adverse pregnancy outcomes. It is crucial to be able to accurately quantify the burden of tobacco exposure on both the mother and fetus to have better measures of efficacy with interventions being studied.

METHODS This is a descriptive and exploratory study conducted within a randomized controlled trial. Pregnant smoking and non-smoking women were followed from $\leq 22$ weeks' gestation through delivery with monthly maternal smoking questionnaires, urine cotinine levels, and collection of maternal and infant hair and nail samples, at delivery. Nicotine was extracted and measured ( $\mathrm{ng} / \mathrm{mg}$ ) using high-performance liquid chromatography with electrochemical detection.

RESULTS Forty-six mother-infant dyads (34 pregnant smokers and 12 pregnant non-smokers) had successful completion of maternal and infant hair and nails samples. The median hair nicotine levels of the smoking mothers and their infants was significantly higher than those of the non-smokers (1.015 vs $0.037 \mathrm{ng}$ / $\mathrm{mg}, \mathrm{p}<0.05$ for the mothers; 0.445 vs $0.080 \mathrm{ng} / \mathrm{mg}, \mathrm{p}<0.01$ for the infants). Similarly, the median nail nicotine levels for smoking mothers and their infants were significantly higher than the non-smokers $(2.130$ vs $0.056 \mathrm{ng} / \mathrm{mg}, \mathrm{p}<0.01$ for the mothers; $0.594 \mathrm{vs} 0.132 \mathrm{ng} / \mathrm{mg}, \mathrm{p}<0.05$ for the infants). We found a moderate but significant correlation between maternal hair and nail nicotine $(\mathrm{r}=0.64, \mathrm{p}<0.001)$, infant hair and nail nicotine $(\mathrm{r}=0.64 ; \mathrm{p}<0.001)$, maternal and infant hair nicotine $(\mathrm{r}=0.61, \mathrm{p}<0.001)$, and maternal and infant nail nicotine levels $(\mathrm{r}=0.58, \mathrm{p}<0.001)$.

ConcLusions Our study shows that both infant hair and nail nicotine levels are valid biomarkers of intrauterine tobacco smoke exposure, and can be used to identify prenatal smoke exposure, correlating well with the level of maternal nicotine exposure.
\end{abstract}

\author{
AFFILIATION \\ 1 Division of Neonatology, \\ Department of Pediatrics, \\ Oregon Health and Science \\ University, Portland, United \\ States \\ 2 Department of Family \\ and Preventive Medicine, \\ University of California-San \\ Diego, California, United \\ States \\ 3 Division of Neuroscience, \\ Oregon National Primate \\ Research Center, Beaverton, \\ United States
}

CORRESPONDENCE TO Mitzi D. Go. Division of Neonatology, Department of Pediatrics, Oregon Health and Science University, 707 SW Gaines Road (CDRC-P), Portland, Oregon 97239, United States. E-mail: gom@ohsu.edu ORCID ID: https://orcid.org/ 0000-0001-7098-3223

\section{KEYWORDS}

infant nail nicotine, infant hair nicotine, intrauterine smoke exposure, maternal hair nicotine, maternal nail nicotine

Received: 29 June 2021 Revised: 21 September 2021 Accepted: 19 October 2021

\section{INTRODUCTION}

Tobacco use remains the single most modifiable cause of adverse pregnancy outcomes, yet, more than $50 \%$ of smokers who become pregnant continue to smoke ${ }^{1,2}$. In addition, for social acceptability reasons, many pregnant women underreport or misrepresent their smoking status ${ }^{3-5}$. The adverse effects of nicotine to the fetus are multiple and well-described, specifically on growth, lung development, asthma, postnatal infections and sudden infant death syndrome (SIDS) ${ }^{3}$. Numerous reports have also established that maternal tobacco smoking during pregnancy is associated with increased risks for adverse maternal conditions (e.g. premature rupture of membranes, abruptio placenta, and placenta previa) and adverse pregnancy outcomes (e.g. neonatal mortality and stillbirth, preterm 
delivery, and SIDS $)^{6-8}$. It is crucial to be able to accurately quantify the burden of tobacco exposure on both the mother and fetus to have efficacious measures in intervention studies.

Numerous maternal and fetal biomarkers have been used to quantify both direct and indirect exposure to nicotine and tobacco exposure, including exhaled carbon monoxide, nicotine or cotinine in urine, saliva, and serum, and urinary 4-(Methylnitrosamino)-1(3-pyridyl)-1-butanol (NNAL), with most reflecting short-term exposure ${ }^{9-11}$. NNAL has a longer half-life but is not present in nicotine replacement products or e-cigarettes making it less useful for those products ${ }^{11}$. Several studies have reported correlations between the hair nicotine levels of mother-infant dyads exposed to secondhand smoke, which was able to reflect a longer duration of exposure (over a period of several months) compared to the other biomarkers ${ }^{12-15}$. Toenail nicotine levels were also reported to capture the longer term overall burden of tobacco smoke exposure in adults ${ }^{5,16}$, while newborn nails have been demonstrated to reflect drug exposure to substances such as caffeine, nicotine, cocaine, benzoylecgonine, morphine, and methadone, during pregnancy ${ }^{17}$. In our randomized placebo-controlled trial of pregnant women who smoke, we reported urine cotinine as our biomarker for maternal smoking status, but to our knowledge, no other study has collected maternal and infant hair and nail samples at delivery alongside a prospective collection of serial maternal smoking questionnaires and urine cotinine levels for the duration of the pregnancy, to determine if nicotine in infant hair and nail could be reliable biomarkers of in-utero exposure to tobacco smoke ${ }^{18}$. Our study aims to demonstrate that infant hair and nail nicotine levels collected shortly after birth correlate with maternal hair and nail nicotine levels and reflect maternal cigarette/nicotine exposure during pregnancy.

\section{METHODS}

This is a descriptive and exploratory study performed within a larger, randomized, double-blind, placebocontrolled trial of pregnant women who were unable to quit smoking, and were randomized to receive vitamin $\mathrm{C}$ ( $500 \mathrm{mg} /$ day) versus placebo prior to 22 weeks gestation with the primary outcome being pulmonary function tests (PFTs) in the newborns after delivery (NCT00632476). A group of pregnant non-smokers and their newborns was studied as a reference group. The protocol was approved by the Institutional Review Boards of the participating institutions, and each pregnant woman provided written informed consent. Study participants were recruited from prenatal clinics at: Oregon Health \& Science University (OHSU) and Providence Maternal Care Clinic in Portland, Oregon; and Vancouver Clinic and PeaceHealth Southwest Medical in Vancouver, Washington; who were aged $\geq 15$ years, current smokers ( $\geq 1$ cigarette/day), with singleton gestation, randomization at $\leq 22$ weeks' gestation age (GA) by last menstrual period (LMP), and had declined smoking cessation attempts as discussed by their physician and study personnel. Exclusion criteria were multiple gestation, major fetal anomalies, current illicit drug or alcohol abuse, continuous daily high dose vitamin C since LMP, insulin dependent diabetic, or history of kidney stones. A group of pregnant nonsmokers, aged $\geq 15$ years, with a singleton gestation $\leq 22$ weeks' GA was recruited as our reference group ${ }^{18}$. The parent study demonstrated that the offspring of pregnant smokers randomized to Vitamin $\mathrm{C}$ had significantly improved PFTs at delivery and had a significant decrease in the incidence of wheeze through one year of age ${ }^{18}$.

A detailed smoking history using questions from a standardized respiratory questionnaire published by the American Thoracic Society for epidemiologic research ${ }^{19}$ was collected at each visit to determine the amount of cigarettes smoked and cigarette exposure, along with other important demographic characteristics. The parent study was started before widespread use of smokeless tobacco products, so these products were not assessed. During subsequent visits, up until the time of delivery, brief smoking cessation counseling ( $<10$ minutes) was performed based on American College of Obstetricians and Gynecologists guidelines ${ }^{20}$, and an interval maternal smoking questionnaire was administered to determine the extent of smoking and/or exposure since the last visit. Urine cotinine was obtained and measured at 24,30 , and 34 weeks of gestation to confirm tobacco smoke exposure reported in the questionnaires ${ }^{21}$.

Samples of maternal hair and nail were obtained at the time of delivery, and neonatal hair was obtained within 48 hours after delivery. Because of innate difficulties in obtaining infant nail samples 
(inadequate amount for 1 collection), mothers were also instructed to collect infant nail clippings for approximately the first 2-3 months of life and return them for nicotine analysis. Hair and nail samples were stored in small sealed plastic bags, and sent to the biochemical laboratory at Wellington Hospital (New Zealand), with nicotine extracted and measured (ng/ $\mathrm{mg}$ ) using high-performance liquid chromatography (HPLC) with electrochemical detection method as described and validated by Mahoney \& Al-Delaimy ${ }^{22}$ and by Al-Delaimy \& Mahoney ${ }^{16}$. As described previously, hair and nail samples were washed with dichloromethane to remove environmental nicotine then digested with $1 \mathrm{M} \mathrm{NaOH}$ prior to HPLC separation $^{16,22}$. Laboratory staff were blinded to the smoking status of the maternal/neonatal samples. To reduce random error, samples were run in duplicate when there were enough samples ( $69 \%$ of hair samples, and $24 \%$ of nail samples).

\section{Specimen collection}

1. Hair: About 10-20 strands of hair were cut as closely as possible to the scalp at the occipital area for both maternal and neonatal samples, with the proximal end marked and the sample tied with a string and stored at room temperature in a sealed paper envelope.

2. Nails: Fingernails were clipped and stored in a small static-free plastic zip bag at room temperature. At least $20 \mathrm{mg}$ of nail samples were collected for every participant. For the newborn infant, to approximate the minimum amount of nail samples needed for analysis, fingernail and toenail clippings were collected for the first 3 months of life by the mother and placed in a small static-free plastic zip bag. This was either mailed back to the study team in a sealed, self-addressed pre-stamped envelope that was provided to them or returned in person during a postnatal visit.

3. Maternal urine was collected at enrollment and subsequent study visits (if available), and cotinine levels were analyzed using a chemiluminescence based immunoassay on an Immulite auto-analyzer (Siemens Health Care Diagnostics) ${ }^{21}$

4. Standard maternal and neonatal data were collected prospectively, and included medical, social and delivery history, including co-morbidities, medications, tobacco use, and alcohol use.
5. Maternal information about hair color, hair treatments and use of hair products were documented, as well as use of nail polish, manicures, or pedicures.

\section{Statistical analysis}

Descriptive statistics were used to characterize maternal and neonatal demographics. We used the t-test to look at the difference in mother-baby hair and nail nicotine levels according to smoking status (smokers vs non-smokers). Raw hair and nail nicotine levels were log transformed using the natural logarithm as the base due to their non-normal distribution. The correlations between the variables and outcomes were performed by the Pearson method, with computed $\mathrm{p}$-values for their significance. All $\mathrm{p}$ values are 2 -sided, and significance was set at $\mathrm{p}<0.05$. Statistical analyses were conducted using $R$ version 4.0.3.

\section{RESULTS}

Forty-six dyads (46) within the cohort had successful completion of all four collection samples (34 pregnant smokers and 12 pregnant non-smokers) for hair and nails. This is an exploratory study started after the main study had already been initiated, so only a subgroup of participants was able to provide all four samples of hair and nails. Table 1 shows the maternal and infant demographics of our study population, with the two groups showing no significant difference in maternal age, mode of delivery, mean gestational age of infant at the time of delivery, and mean birthweight. There is a higher percentage of Medicaid insured mothers who smoke compared to non-smokers. Table 2 summarizes our primary outcome of nicotine levels in hair and nails of the two groups of mother-infant dyads within our cohort. The median hair nicotine level for mothers who smoke was significantly higher than that of the non-smokers ( 1.015 vs $0.037 \mathrm{ng} / \mathrm{mg}$, $\mathrm{p}<0.05)$, while the hair nicotine level of infants born to mothers who smoke was also significantly higher than the infants of the non-smokers ( 0.445 vs 0.080 $\mathrm{ng} / \mathrm{mg}, \mathrm{p}<0.01)$. Similarly, we found that the median nail nicotine levels for mothers who smoke were significantly higher than the non-smokers (2.130 vs $0.056 \mathrm{ng} / \mathrm{mg}, \mathrm{p}<0.01)$ and the median nail nicotine levels of their respective infants reflected the same pattern $(0.594$ vs $0.132 \mathrm{ng} / \mathrm{mg}, \mathrm{p}<0.05)$. 
Table 1. Maternal and infant characteristics of the study cohort ( $\mathrm{N}=46)$

\begin{tabular}{|c|c|c|c|c|}
\hline Characteristics & $\begin{array}{c}\text { Non-smoker } \\
\text { Median or } \\
n(\%)\end{array}$ & $\begin{array}{l}\text { Percentiles* } \\
\text { (Q1; Q3) }\end{array}$ & $\begin{array}{l}\text { Smoker } \\
\text { Median or } \\
n(\%)\end{array}$ & $\begin{array}{l}\text { Percentiles* } \\
\text { (Q1; Q3) }\end{array}$ \\
\hline Total, n & 12 & & 34 & \\
\hline \multicolumn{5}{|l|}{ Maternal } \\
\hline Cigarettes/day & 0 & 0 & 7.29 & $(2 ; 12)$ \\
\hline Maternal age (years) & 26.25 & $(19.75 ; 30.25)$ & 25.85 & $(20 ; 28.75)$ \\
\hline Gravida & 2 & $(1 ; 2)$ & 3.09 & $(1 ; 5)$ \\
\hline Parity & 0.58 & $(0 ; 1)$ & 1.323 & $(0 ; 2)$ \\
\hline Insurance (Medicaid vs gov't insurance) & $6(50.0)$ & & $32(94.0)$ & \\
\hline Maternal asthma & $2(17.0)$ & & $7(20.0)$ & \\
\hline \multicolumn{5}{|l|}{ Race } \\
\hline White & $10(83.3)$ & & $27(79.4)$ & \\
\hline Black & $1(8.3)$ & & $6(17.6)$ & \\
\hline American Indian & 0 & & $1(3.0)$ & \\
\hline Asian & $1(8.3)$ & & 0 & \\
\hline \multicolumn{5}{|l|}{ Maternal education level } \\
\hline Some grade school & $1(8.3)$ & & $6(17.6)$ & \\
\hline Some high school & $6(50.0)$ & & $21(61.8)$ & \\
\hline Some college & $2(16.7)$ & & $7(20.6)$ & \\
\hline Some postgraduate & $3(25.0)$ & & 0 & \\
\hline \multicolumn{5}{|l|}{ Other maternal and infant } \\
\hline Maternal hair treated/dyed/bleached & $1(8.0)$ & & $6(18.0)$ & \\
\hline Maternal nails polished/manicure/pedicure & $3(25.0)$ & & $6(18.0)$ & \\
\hline Delivery mode (cesarean section) & $3(25.0)$ & & $8(24.0)$ & \\
\hline Infant GA (weeks) & 38.3 & $(37.5 ; 39.9)$ & 38.9 & $(38.1 ; 40)$ \\
\hline Birthweight (g) & 3175 & $(2765 ; 3739)$ & 3326 & $(2956 ; 3536)$ \\
\hline \multicolumn{5}{|l|}{ Sex } \\
\hline Male & $4(33.0)$ & & $18(53.0)$ & \\
\hline Female & $8(67.0)$ & & $16(47.0)$ & \\
\hline
\end{tabular}

* $01=25$ th, 03=75th percentile. Subjects recruited at: Oregon Health \& Science University (OHSU) and Providence Maternal Care Clinic in Portland, Oregon; and Vancouver Clinic and PeaceHealth Southwest Medical in Vancouver, Washington.

Table 2. Nicotine levels in hair and nails of pregnant smokers and non-smokers, and of their offspring, collected at delivery $(\mathrm{N}=46)$

\begin{tabular}{|c|c|c|c|c|c|}
\hline Biomarker & $\begin{array}{c}\text { Non-smoker and } \\
\text { infant dyad } \\
\text { Median }\end{array}$ & $\begin{array}{l}\text { Percentiles* } \\
\text { (Q1; Q3) }\end{array}$ & $\begin{array}{l}\text { Smoker and } \\
\text { infant dyad } \\
\text { Median }\end{array}$ & $\begin{array}{l}\text { Percentiles* } \\
\text { (Q1; Q3) }\end{array}$ & $p$ \\
\hline Total, n & 12 & & 34 & & \\
\hline Cigarettes/day & 0 & & 7 & $(2 ; 12)$ & \\
\hline Urine cotinine (ng/mL) & 10 & $(10 ; 14.42)$ & 3019 & $(861 ; 6712)$ & \\
\hline Maternal hair nicotine $(\mathrm{ng} / \mathrm{mg})$ & 0.037 & $(0.01 ; 0.09)$ & 1.015 & $(0.56 ; 2.76)$ & $<0.05$ \\
\hline Infant hair nicotine $(\mathrm{ng} / \mathrm{mg})$ & 0.080 & $(0.03 ; 0.16)$ & 0.445 & $(0.19 ; 0.69)$ & $<0.01$ \\
\hline Maternal nail nicotine $(\mathrm{ng} / \mathrm{mg})$ & 0.056 & $(0.02 ; 0.08)$ & 2.130 & $(0.48 ; 4.14)$ & $<0.01$ \\
\hline Infant nail nicotine (ng/mg) & 0.133 & $(0.03 ; 0.37)$ & 0.594 & $(0.24 ; 1.17)$ & $<0.05$ \\
\hline
\end{tabular}

${ }^{*} 01=25$ th, $Q 3=75$ th percentile. Urine cotinine based on 1 to 3 occasions for each participant ( 2 missing non-smokers, 7 missing from smokers). Samples collected from subjects recruited at: Oregon Health \& Science University (OHSU) and Providence Maternal Care Clinic in Portland, Oregon; and Vancouver Clinic and PeaceHealth Southwest Medical in Vancouver, Washington. 
Figure 1. Correlation between infant hair and nail nicotine levels $(n=46, r=0.64, p<0.001)$

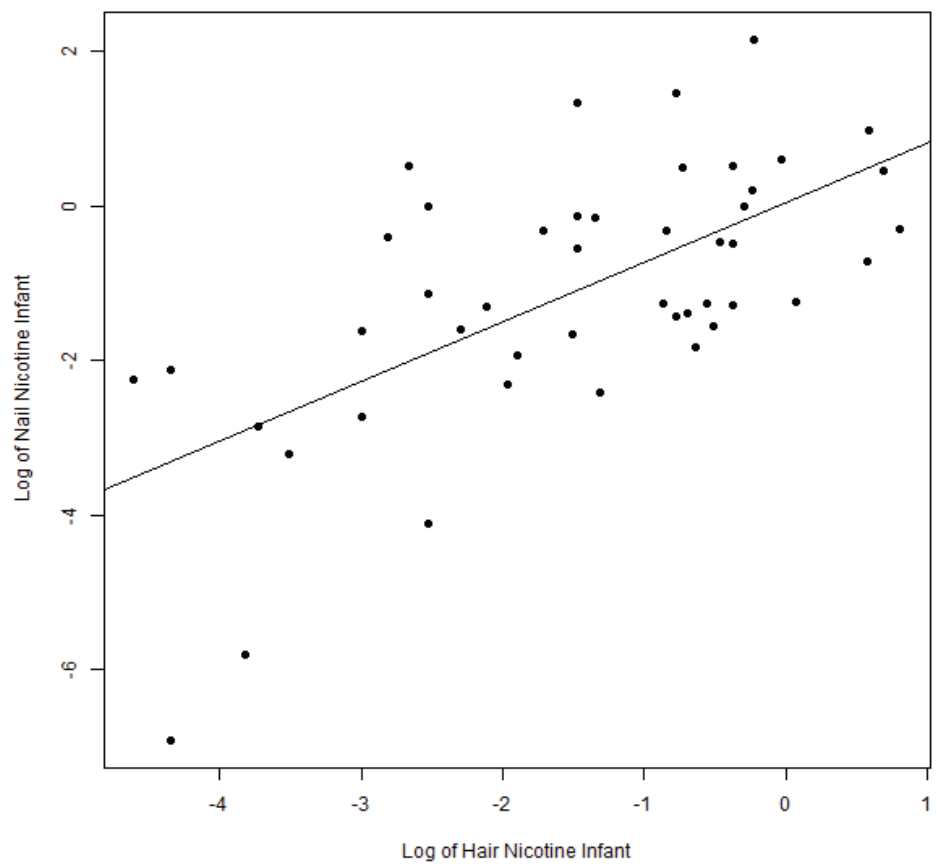

Figure 2. Correlation between maternal and infant nail nicotine levels $(n=46, r=0.58, p<0.001)$

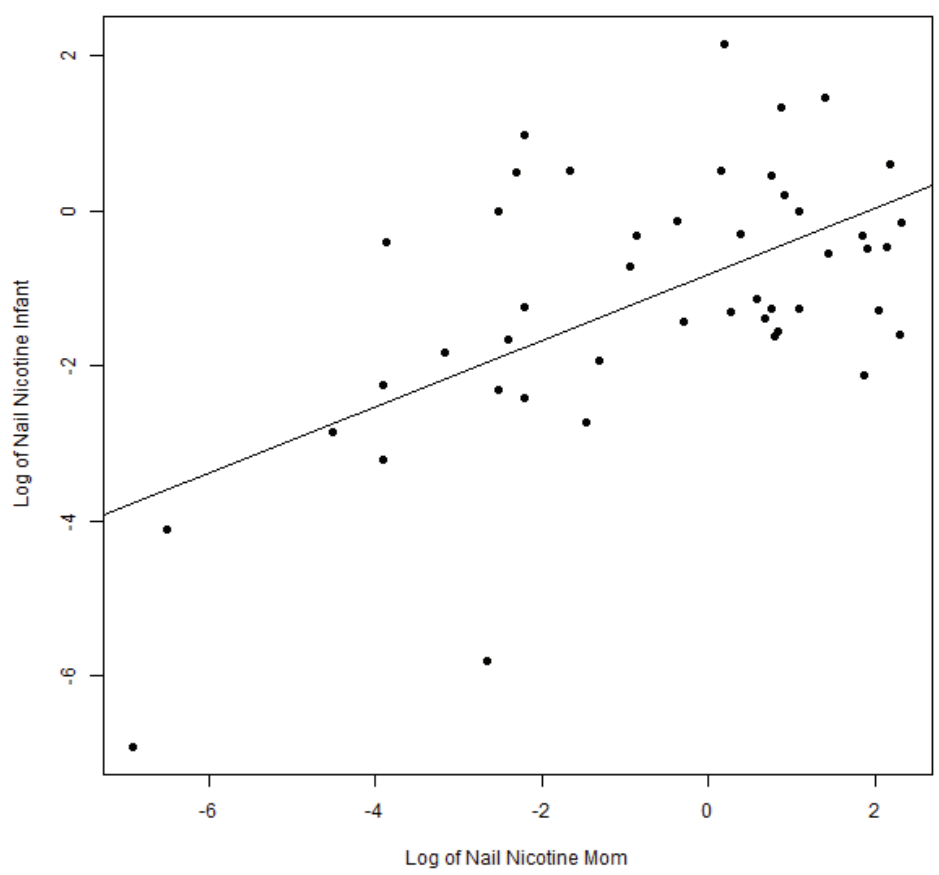

We found expected correlations between maternal hair and nail nicotine levels with cigarettes per day $(\mathrm{r}=0.60$ and $\mathrm{r}=0.53 ; \mathrm{p}<0.001$, respectively $)$, as well as maternal hair and nail nicotine levels with average urine cotinine during the pregnancy $(\mathrm{r}=0.82$ and $\mathrm{r}=0.6 ; \mathrm{p}<0.001)$. There are strong correlations between maternal hair and maternal nail nicotine $(\mathrm{r}=0.64, \mathrm{p}<0.001)$ as well as between infant hair and infant nail nicotine levels $(r=0.64 ; \mathrm{p}<0.001)$ (Figure 1 ). The correlations between maternal and infant hair 
nicotine levels $(\mathrm{r}=0.61, \mathrm{p}<0.001)$ as well as maternal and infant nail nicotine levels $(\mathrm{r}=0.58, \mathrm{p}<0.001)$ (Figure 2), were also significant.

\section{DISCUSSION}

The levels of nicotine, its major metabolite cotinine, and tobacco-specific nitrosamines have been used as biomarkers for the quantitative assessment of tobacco smoke exposure in numerous studies ${ }^{10,12,14,23,24}$. Cotinine, a major metabolite of nicotine, has been extensively measured in urine and plasma as a biomarker of firsthand and secondhand smoke exposure. However, these measurements can only reflect the last few days of tobacco smoke exposure at best, despite cotinine having a longer half-life than nicotine in these samples. Hair nicotine have been shown to capture chronic exposure in adults and children, while toenail nicotine has more recently been demonstrated to do the same in adults. The advantages of hair and nail nicotine levels over plasma and urine in providing better information on longterm or chronic exposure, and the non-invasive nature of specimen collection make them ideal biomarkers of exposure for infants and children. These samples can also be stored and analyzed years later, without requiring sophisticated storage methodologies or technology.

Analysis of maternal and neonatal hair as a biological marker for nicotine exposure during pregnancy has been studied by Klein and Koren ${ }^{24}$ and Pichini et al. ${ }^{25}$. In the study by Klein and Koren ${ }^{24}$, they found a significant correlation between maternal and neonatal hair nicotine levels of 94 dyads, with maternal levels being significantly higher than newborn levels across 3 group classifications (active smokers, passive smokers, non-smokers). In the study by Pichini et al. ${ }^{25}$, nicotine could not be quantified in $30.7 \%$ of newborn hair samples, and they found no correlation between maternal hair nicotine and newborn hair nicotine levels. In their study, nicotine concentration in hair of newborns from non-exposed non-smokers and exposed non-smokers were also similar, a contrast to the observation by Klein and Koren ${ }^{24}$. The two studies used different assay methodologies, with the former using radioimmunoassay technique, while the latter used gas chromatography ${ }^{24,25}$. Our study used reversephase high performance liquid chromatography with electrochemical detection, a methodology developed and validated by Mahoney \& Al-Delaimy ${ }^{22}$, Al-Delaimy $\&$ Mahoney $^{16}$, and by Al-Delaimy \& Willett ${ }^{5}$, and despite using a different assay and result values, our study findings are more consistent with the findings of Klein and Koren ${ }^{24}$, where we found a strong correlation between maternal and newborn hair nicotine levels, demonstrating that newborn hair nicotine can be a valuable predictor of fetal exposure to cigarette smoke. An unexpected observation in our dataset was that in our non-smoking group, the median newborn hair and nail nicotine levels appeared to be higher than the maternal levels. However, these levels were still significantly lower than the respective levels in the babies of smokers. This could be a marker of secondhand smoke exposure that was not captured by the respiratory questionnaires administered during pregnancy.

It is also interesting to note that we found that the levels of nicotine detected in the nails were higher than those noted in the hair among both our infant and maternal smoking and non-smoking samples, in contrast to the findings of Al-Delaimy \& Mahoney ${ }^{16}$ and Al-Delaimy \& Willett ${ }^{5}$, whose studies have shown lower levels of nicotine in nails than in hair in adults. Human nails have been used to monitor excessive exposure to elements for many years, and there is an abundance of literature that has reported the use of nail analysis in postmortem detection of drugs of abuse, drug testing in the workplace, and drug screening to detect prenatal exposure, even though further studies are needed for correct interpretation of the data obtained ${ }^{26,27}$. Palmeri et al. ${ }^{26}$, have shown that drugs are incorporated into nails by a double mechanism: 1) deposition into the root of the growing nail via the blood flow in the nail matrix; and 2 ) incorporation via the nail bed during growth from the lunula to the beginning of the free margin. Unlike hair, nails are not influenced by melanin content, which is a potential confounder for nicotine deposition ${ }^{28-30}$. We therefore speculate that our results may reflect the fact that the deposition of nicotine in the nails is less affected by acute and short-term environmental changes applied to the human body compared to hair, which can be altered or influenced by race/ethnicity, type of hair, hair color, or treatments done to the hair (bleaching, straightening, permanent, etc.) ${ }^{31}$. Our findings that newborn nail nicotine levels were higher than newborn hair nicotine levels could represent the 
susceptibility of nails to nicotine deposition prenatally, as the formation of nail beds occur earlier in the fetus (at around 8-10 weeks' gestation), which do not undergo shedding in-utero compared to hair, and are shed into the amniotic fluid prior to delivery ${ }^{32}$.

This is the first study to quantify and demonstrate that newborn nail nicotine levels can be used to assess in-utero tobacco smoke exposure. Babies born to mothers that were unable to quit smoking during pregnancy had significantly higher levels of nail nicotine levels than those born to non-smokers. In addition, we demonstrated that the nail nicotine levels had a strong correlation with other biomarkers of nicotine exposure including infant hair nicotine levels and maternal hair and nail nicotine levels at delivery. Baby nail nicotine levels also had a significant correlation $(\mathrm{r}=0.62 ; \mathrm{p}<0.001)$ with maternal urine cotinine levels obtained during pregnancy, a more standard measure of nicotine exposure, while they had a moderate correlation $(\mathrm{r}=0.44 ; \mathrm{p}<0.001)$ with maternal reported cigarettes per day during pregnancy. To our knowledge, no other study had investigated nail nicotine in newborns in relation to maternal nail nicotine levels and smoking status during pregnancy. Al-Delaimy and Willett ${ }^{5,33}$ demonstrated that toenail nicotine levels captured the tobacco smoke exposure in a large cohort of women that participated in the Nurses' Health Study and were a strong predictor of lung cancer in a large male cohort within the Health Professionals Follow-up Study. Mari et al..$^{22}$ reported that nicotine was detected in newborn nails, but did not have maternal data to correlate with in-utero exposure and nicotine levels were not quantified.

\section{Strengths and limitations}

The strength of our study lies in the prospective collection of multiple biomarkers (maternal urine cotinine, maternal and newborn hair and nail nicotine) accompanied by serial respiratory questionnaires, collected during pregnancy and postnatally from the same mother-infant dyads. Nicotine extraction and measurements were also performed at a wellestablished and published laboratory with rigorous testing procedures. Nicotine also has the advantage of being the primary bioactive compound and present in all tobacco products, e-cigarette and nicotine replacement products. A limitation would be a possible underestimation of nicotine exposure from self-reported questionnaires. Pregnant women when asked to provide information on smoking habits, often underreport actual behaviors because of social pressures and possible feelings of guilt, if they think it is unhealthy for the baby and themselves. Also, the non-smokers in the cohort included former smokers who had quit prior to pregnancy and may have continued to be passively exposed to tobacco smoke but not captured in our study questionnaire. We also have a relatively small sample size due to difficulty obtaining hair and nail samples from the newborn infant. We found that mothers would consent to the taking of their own samples but often hesitated in agreeing for hair to be cut from their newborn for aesthetic or cultural reasons. We did not analyze maternal urine for tobacco-specific nitrosamines, which have a relatively long half-life and are also specific to conventional tobacco products as they are not present in e-cigarettes and nicotine-replacement products ${ }^{10}$. However, despite these limitations, our study shows that newborn nail nicotine levels correlate well with maternal nail nicotine levels, with levels significantly higher for the smoking group compared to the non-smokers, and newborn levels showing a strong correlation with maternal levels.

\section{CONCLUSIONS}

Our study shows that both infant hair and nail nicotine levels are valid biomarkers of intrauterine tobacco smoke exposure, and can be used to identify prenatal smoke exposure, correlating well with the level of maternal nicotine exposure. Measuring nail nicotine levels in newborns provides an advantage over hair nicotine levels in situations where newborn hair is unavailable or inadequate, and may better reflect prenatal smoke exposure due to the earlier development of nail beds compared to hair follicles in the fetus. This finding has many possible applications in clinical and research settings to determine causes of respiratory illnesses of the newborn, low birthweight, or longer term childhood illnesses.

\section{REFERENCES}

1. Filion KB, Abenhaim HA, Mottillo S, et al. The effect of smoking cessation counselling in pregnant women: a meta-analysis of randomised controlled trials. BJOG. 2011;118(12):14221428. doi:10.1111/j.1471-0528.2011.03065.x

2. Schneider S, Huy C, Schütz J, Diehl K. Smoking cessation during pregnancy: a systematic literature review. Drug Alcohol Rev. 


\section{0;29(1):81-90. doi:10.1111/j.1465-3362.2009.00098.x}

3. U.S. Department of Health and Human Services. The Health Consequences of Smoking: 50 Years of Progress. A Report of the Surgeon General. U.S. Department of Health and Human Services, Centers for Disease Control and Prevention, National Center for Chronic Disease Prevention and Health Promotion, Office on Smoking and Health; 2014. Accessed September 21, 2021. https:// www.ncbi.nlm.nih.gov/books/NBK179276/

4. Al-Delaimy WK. Hair as a biomarker for exposure to tobacco smoke. Tob Control. 2002;11(3):176-182. doi:10.1136/tc.11.3.176

5. Al-Delaimy WK, Willett WC. Measurement of tobacco smoke exposure: comparison of toenail nicotine biomarkers and selfreports. Cancer Epidemiol Biomarkers Prev. 2008;17(5):12551261. doi:10.1158/1055-9965.EPI-07-2695

6. Ernst M, Moolchan ET, Robinson ML. Behavioral and neural consequences of prenatal exposure to nicotine. J Am Acad Child Adolesc Psychiatry. 2001;40(6):630-641. doi:10.1097/00004583-200106000-00007

7. Centers for Disease Control and Prevention. Smokingattributable mortality, years of potential life lost, and productivity losses--United States, 2000-2004. MMWR Morb Mortal Wkly Rep. 2008;57(45):1226-1228. Accessed September 21, 2021. https://www.cdc.gov/ $\mathrm{mmwr} /$ preview/mmwrhtml/mm5745a3.htm

8. Lee J, Lee DR, Lee DH, Paek YJ, Lee WC. Influence of maternal environmental tobacco smoke exposure assessed by hair nicotine levels on birth weight. Asian Pac J Cancer Prev. 2015;16(7):3029-3034. doi:10.7314/apjcp.2015.16.7.3029

9. Avila-Tang E, Al-Delaimy WK, Ashley DL, et al. Assessing secondhand smoke using biological markers. Tob Control. 2013;22(3):164-171. doi:10.1136/tobaccocontrol-2011-050298

10. Loukopoulou AN, Vardavas CI, Farmakides G, et al. Counselling for smoking cessation during pregnancy reduces tobacco-specific nitrosamine (NNAL) concentrations: A randomized controlled trial. Eur J Midwifery. 2018;2:14. doi:10.18332/ejm/99546

11. Benowitz NL, Bernert JT, Foulds J, et al. Biochemical Verification of Tobacco Use and Abstinence: 2019 Update. Nicotine Tob Res. 2020;22(7):1086-1097. doi:10.1093/ntr/ntz132

12. Eliopoulos C, Klein J, Phan MK, et al. Hair concentrations of nicotine and cotinine in women and their newborn infants. JAMA. 1994;271(8):621-623. Accessed September 21, 2021. https://jamanetwork.com/journals/ jama/article-abstract/365789

13. Ashford KB, Hahn E, Hall L, Rayens MK, Noland M, Collins R. Measuring prenatal secondhand smoke exposure in mother-baby couplets. Nicotine Tob Res. 2010;12(2):127-135. doi:10.1093/ntr/ntp185

14. Florescu A, Ferrence R, Einarson TR, et al. Reference values for hair cotinine as a biomarker of active and passive smoking in women of reproductive age, pregnant women, children, and neonates: systematic review and meta-analysis. Ther Drug Monit. 2007;29(4):437-446. doi:10.1097/FTD.0b013e318074df6e

15. Li Z, Li Z, Zhang J, et al. Using nicotine in scalp hair to assess maternal passive exposure to tobacco smoke. Environ Pollut. 2017;222:276-282. doi:10.1016/j.envpol.2016.12.044

16. Al-Delaimy WK, Mahoney GN, Speizer FE, Willett WC. Toenail nicotine levels as a biomarker of tobacco smoke exposure. Cancer Epidemiol Biomarkers Prev. 2002;11(11):1400-1404. Accessed September 21, 2021. https://cebp.aacrjournals.org/content/11/11/1400.long

17. Mari F, Politi L, Bertol E. Nails of newborns in monitoring drug exposure during pregnancy. Forensic Sci Int. 2008;179(2-3):176-180. doi:10.1016/j.forsciint.2008.06.001

18. McEvoy CT, Schilling D, Clay N, et al. Vitamin C supplementation for pregnant smoking women and pulmonary function in their newborn infants: a randomized clinical trial. JAMA. 2014;311(20):20742082. doi:10.1001/jama.2014.5217

19. Ferris BG. Epidemiology Standardization Project (American Thoracic Society). Am Rev Respir Dis. 1978;118(6 Pt 2):1-120. Accessed September 21, 2021. https://pubmed.ncbi.nlm.nih.gov/742764/

20. The American College of Obstetricians and Gynecologists. Committee opinion no. 471: Smoking cessation during pregnancy. Obstet Gynecol. 2010;116(5):1241-1244. doi:10.1097/AOG.0b013e3182004fcd

21. Benowitz NL, Hukkanen J, Jacob P 3rd. Nicotine chemistry, metabolism, kinetics and biomarkers. Handb Exp Pharmacol. 2009;(192):29-60. doi:10.1007/978-3-540-69248-5_2

22. Mahoney GN, Al-Delaimy W. Measurement of nicotine in hair by reversed-phase high-performance liquid chromatography with electrochemical detection. J Chromatogr B Biomed Sci Appl. 2001;753(2):179-187. doi:10.1016/s0378-4347(00)00540-5

23. Almeida ND, Koren G, Platt RW, Kramer MS. Hair biomarkers as measures of maternal tobacco smoke exposure and predictors of fetal growth. Nicotine Tob Res. 2011;13(5):328-335. doi:10.1093/ntr/ntq259

24. Klein J, Koren G. Hair analysis--a biological marker for passive smoking in pregnancy and childhood. Hum Exp Toxicol. 1999;18(4):279-282. doi:10.1191/096032799678840048

25. Pichini S, Garcia-Algar O, Munoz L, et al. Assessment of chronic exposure to cigarette smoke and its change during pregnancy by segmental analysis of maternal hair nicotine. Journal of Exposure Analysis and Environmental Epidemiology. 2003;13:144-151. doi:10.1038/sj.jea.7500264

26. Palmeri A, Pichini S, Pacifici R, Zuccaro P, Lopez A. Drugs in nails: physiology, pharmacokinetics and forensic toxicology. Clin Pharmacokinet. 2000;38(2):95-110. doi:10.2165/00003088-200038020-00001 
27. Krumbiegel F, Hastedt M, Westendorf L, et al. The use of nails as an alternative matrix for the long-term detection of previous drug intake: validation of sensitive UHPLCMS/MS methods for the quantification of 76 substances and comparison of analytical results for drugs in nail and hair samples. Forensic Sci Med Pathol. 2016;12(4):416434. doi:10.1007/s12024-016-9801-1

28. Dehn DL, Claffey DJ, Duncan MW, Ruth JA. Nicotine and cotinine adducts of a melanin intermediate demonstrated by matrix-assisted laser desorption/ionization timeof-flight mass spectrometry. Chem Res Toxicol. 2001;14(3):275-279. doi:10.1021/tx000205l

29. Claffey DJ, Stout PR, Ruth JA. 3H-nicotine, $3 \mathrm{H}$-flunitrazepam, and $3 \mathrm{H}$-cocaine incorporation into melanin: a model for the examination of drug-melanin interactions. J Anal Toxicol. 2001;25(7):607-611. doi:10.1093/jat/25.7.607

30. Yerger VB, Malone RE. Melanin and nicotine: A review of the literature. Nicotine Tob Res. 2006;8(4):487-498. doi:10.1080/14622200600790039

31. Apelberg BJ, Hepp LM, Avila-Tang E, et al. Racial differences in hair nicotine concentrations among smokers. Nicotine Tob Res. 2012;14(8):933-941. doi:10.1093/ntr/ntr311

32. Eichenfield LF, Frieden IJ, Mathes E, Zaenglein A. Neonatal and Infant Dermatology. 3rd ed. Elsevier; 2014.

33. Al-Delaimy WK, Willett WC. Toenail nicotine level as a novel biomarker for lung cancer risk. Am J Epidemiol. 2011;173(7):822-828. doi:10.1093/aje/kwq446
CONFLICTS OF INTEREST

The authors have each completed and submitted an ICMJE form for disclosure of potential conflicts of interest. The authors declare that they have no competing interests, financial or otherwise, related to the current work. All the authors report that since the initial planning of the work, payments were made to the institution: NHLBI (K23 HL080231), Office of Dietary Supplements and NIH (UH3 OD023288), Oregon Clinical Translational Research Institute funded by the National Center for Advancing Translational Sciences (UL1TR000128). M.D. Go also reports that in the past 36 months was American Thoracic Society Assembly on Pediatrics Co-chair of Journal Club Webinar Committee. E.R. Spindel reports that in the past 36 months payments were made to the institution (P51 OD011092565). C.T. McEvoy reports that in the past 36 months she was an UpToDate Peer Reviewer in BPD prevention, management, postnatal corticosteroid use.

\section{FUNDING}

This work was supported by National Heart, Lung, and Blood Institute (NHLBI) (Grant K23 HL080231) with co-funding from Office of Dietary Supplements and NIH (UH3 OD023288). Additional support was from the Oregon Clinical Translational Research Institute funded by the National Center for Advancing Translational Sciences (UL1TR000128).

\section{ETHICAL APPROVAL AND INFORMED CONSENT}

Ethical approval was obtained from the Institutional Review Boards of the participating institutions (IRB00002893; August 27, 2004). Study participants were recruited from prenatal clinics at: Oregon Health \&t Science University (OHSU) and Providence Maternal Care Clinic in Portland, Oregon, and Vancouver Clinic and PeaceHealth Southwest Medical in Vancouver, Washington. Each pregnant woman provided written informed consent.

\section{DATA AVAILABILITY}

The data supporting this research are available from the authors on reasonable request.

PROVENANCE AND PEER REVIEW

Not commissioned; externally peer reviewed. 